\title{
Spatial and temporal characteristics of poloidal waves in the terrestrial plasmasphere: a CLUSTER case study
}

\author{
S. Schäfer ${ }^{1}$, K. H. Glassmeier ${ }^{1}$, P. T. I. Eriksson ${ }^{2}$, V. Pierrard ${ }^{3}$, K. H. Fornaçon ${ }^{1}$, and L. G. Blomberg ${ }^{2}$ \\ ${ }^{1}$ Institut für Geophysik und extraterrestrische Physik, TU Braunschweig, Germany \\ ${ }^{2}$ Space and Plasma Physics, School of Electrical Engineering, Royal Institute of Technology Stockholm, Sweden \\ ${ }^{3}$ Belgian Institute for Space Aeronomy, Brussels, Belgium
}

Received: 20 December 2006 - Revised: 2 April 2007 - Accepted: 13 April 2007 - Published: 8 May 2007

\begin{abstract}
Oscillating magnetic field lines are frequently observed by spacecraft in the terrestrial and other planetary magnetospheres. The CLUSTER mission is a very suitable tool to further study these Alfvén waves as the four CLUSTER spacecraft provide for an opportunity to separate spatial and temporal structures in the terrestrial magnetosphere. Using a large scaled configuration formed by the four spacecraft we are able to detect a poloidal Ultra-Low-Frequency (ULF) pulsation of the magnetic and electric field in order to analyze its temporal and spatial structures. For this purpose the measurements are transformed into a specific field line related coordinate system to investigate their specific amplitude pattern depending on the path of the CLUSTER spacecraft across oscillating field lines. These measurements are then compared with modeled spacecraft observations across a localized poloidal wave resonator in the dayside plasmasphere. A detailed investigation of theoretically expected poloidal eigenfrequencies allows us to specify the observed $16 \mathrm{mHz}$ pulsation as a third harmonic oscillation. Based on this we perform a case study providing a clear identification of wave properties such as an spatial scale structure of about $0.67 R_{E}$, the azimuthal wave number $m \approx 30$, temporal evolution, and energy transport in the detected ULF pulsations.
\end{abstract}

Keywords. Magnetospheric physics (MHD waves and instabilities; Plasmasphere) - Space plasma physics (Waves and instabilities)

\section{Introduction}

Next to processes such as magnetic reconnection or collisionless shock waves the process of resonant mode coupling or magnetospheric field line resonance is a physical process

Correspondence to: S. Schäfer

(seb.schaefer@tu-bs.de) whose knowledge we owe ground-based and in-situ observation of space plasmas using spacecraft observations. The theoretical basis for resonant coupling processes was established by Tamao (1965), Chen and Hasegawa (1974), and Southwood (1974) who were the first to provide a theoretical framework to understand the resonant coupling between compressional waves with small azimuthal wave number $m$ and Alfvén waves in an inhomogeneous plasma.

The physical resonant mode coupling can be understood considering electric current continuity. From Maxwell's equations the following wave equation is derived for lowfrequency waves:

$\nabla \times \nabla \times \boldsymbol{E}+\mu_{0} \frac{\partial \boldsymbol{j}}{\partial t}=0$.

Here $\boldsymbol{E}$ denotes the wave electric field, and $\boldsymbol{j}$ is the electric current density driving the wave. A magnetohydrodynamic wave in a homogeneous plasma carries polarization currents

$\boldsymbol{j}_{\boldsymbol{p}}=\frac{1}{\mu_{0} v_{A}^{2}} \frac{d \boldsymbol{E}}{d t}$,

where $v_{A}=B_{0} / \mu_{0} \rho_{0}$ defines the Alfvén velocity and $\boldsymbol{B}_{\mathbf{0}}$ and $\rho_{0}$ denote the background magnetic field and the plasma mass density, respectively. For an Alfvén mode this polarization current is curl-free in a plane whose normal is the background magnetic field $\boldsymbol{B}_{0}$. The fast mode carries a sourcefree polarization current. Considering a fast mode type perturbation with its source-free current in an inhomogeneous medium with spatially changing Alfvén velocity current continuity requires the fast mode carried current to be partially closed via field-aligned currents driving Alfvénic perturbations carried by a curl-free polarization current system. If the phase velocity component of the fast mode parallel to the background magnetic field is equal to the local Alfvén velocity both waves have ample opportunity to exchange their energy; resonance takes place.

Published by Copernicus GmbH on behalf of the European Geosciences Union. 
Observational evidence for the existence of such resonances was provided by e.g. Samson et al. (1971) and Walker et al. (1979) using ground-based and ionospheric observations, respectively. Also spacecraft observations provide some evidence for the existence of field line resonances in the terrestrial magnetosphere (e.g. Engebretson and Cahill, 1981; Engebretson et al., 1986). The azimuthal variation of these observed resonances was found to be rather small; typical azimuthal wave numbers are of the order of $m=5$ (e.g. Olson and Rostoker, 1978). In the radial direction widths of standing Alfvén waves are observed between $0.2 R_{E}$ and $1.6 R_{E}$ (e.g. Walker et al., 1979; Singer et al., 1982; Mitchell et al., 1990).

The source for the primary fast mode wave is in many occasions a Kelvin-Helmholtz instability of the magnetopause (e.g. Engebretson et al., 1997). A dominating wave damping mechanism for the coupled Alfvén mode is Joule heating in the ionosphere (e.g. Glassmeier et al., 1984).

However, many ULF pulsation observations, though interpreted as field line resonances, lag a definite proof of their resonant character as single spacecraft observations do not allow to demonstrate the spatial localization of the wave amplitude and the typical $180^{\circ}$ phase change of the toroidal component in radial direction. What is observed is usually a spatial localization in the radial or poloidal component, not the toroidal component (e.g. Singer et al., 1979, 1982; Singer, 1982; Cramm et al., 2000). Furthermore, such poloidally localized waves exhibit large azimuthal wave numbers $m \approx 50-150$. For such large $m$-value ULF pulsations the field line resonance mechanism is a rather inefficient one (e.g. Kivelson and Southwood, 1986; Lee and Lysak, 1990) and cannot be used as an explanation to understand these poloidal resonances. Leonovich and Mazur (1990) give a simple reason for this: in the limit $m \rightarrow 0$ the azimuthal component of the wave vector $k_{\phi}$ is equal to infinity. But also the radial component $k_{r}$ tends to infinity in case of an ideal resonance. Because of $\nabla \cdot \boldsymbol{B}=0$ the direction of the transverse magnetic field component is not defined.

The question thus arises on how to understand the spatial structure of poloidal ULF pulsations with large $m$ values. Observations from single spacecraft have only a limited value in providing the necessary experimental background to tackle this question. Due to spacecraft motion a separation between spatial and temporal variations is not possible. Temporal variations can be introduced by the dynamics of the wave source itself. They also reflect the described coupling processes which take a finite time to reach a steady state situation. Phase mixing as discussed in more detail by e.g. Mann and Wright (1995) thus causes significant temporal variations and polarization changes which cannot be resolved by single spacecraft measurements.

Here the four CLUSTER spacecraft (Escoubet et al., 1997) represent a very suitable tool to analyze magnetospheric ULF pulsations as shown in a recent study of poloidal ULF pulsations by Eriksson et al. (2005b). These spacecraft move through the magnetosphere on polar orbits forming various scaled configurations with distances between $0.016 R_{E}$ and $2.0 R_{E}$. Compared to previous missions with one or two spacecraft the advantage of the CLUSTER mission consists in the availability of four measurement points either close to each other within the same region of interest or located in different regions. Due to its polar orbit the region close to the plasmapause is of particular interest. Here plasma conditions are more complex than in the outer region of the magnetosphere as steep plasma density gradients, finite plasma beta, field line curvature, and the ring current may influence the ULF pulsation wave field. Also, as the plasmapause is located deep in the magnetosphere wave sources are not necessarily associated with processes at the magnetopause. Rather local populations of energetic particles are generally accepted as a wave source of poloidal oscillations (Ozeke and Mann, 2001) leading to plasma instabilities such as the drift mirror (Hasegawa, 1969) and the bounce instabilitiy (Southwood et al., 1969; Southwood and Kivelson, 1982).

A suitable theoretical framework to interpret the spatial structure of poloidal oscillations with large $m$-values has been presented by e.g. Leonovich and Mazur (1990). Some of their results can be understood again considering the different currents associated with the restoring forces influencing the wave. For example, the influence of a finite plasma $\beta$ can be incorporated in Eq. (1) by a diamagnetic current

$j_{\beta}=\frac{\nabla \boldsymbol{P} \times \boldsymbol{B}_{\mathbf{0}}}{B_{0}^{2}}$.

Magnetic field line curvature introduces curvature currents $\boldsymbol{j}_{\boldsymbol{c}}$. If the background plasma carries a significant electric current $J$ as observed in the ring current region a further current,

$\boldsymbol{j}_{\boldsymbol{J}}=\frac{(\boldsymbol{J} \times \boldsymbol{b}) \times \boldsymbol{B}_{\mathbf{0}}}{B_{0}^{2}}$,

needs to be considered when solving Eq. (1); here $\boldsymbol{b}$ denotes the magnetic field perturbation vector. This current describes forces associated with work done by the perturbed plasma against the current-carrying background.

Each of these additional currents influences wave dispersion and propagation velocity of ULF waves observed in the magnetosphere. Based on this Leonovich and Mazur (1990, 1993, 1995) and Klimushkin (1998a) have derived a rather general theoretical framework to describe the dispersion characteristics of ULF waves in more complex background plasma situations. Especially the curvature currents are important as they introduce transverse dispersion causing large- $m$ waves to propagate in radial direction. Wave propagation inwards is hampered by the increasing plasma mass density and magnetic field strength; the inner magnetosphere/plasmasphere becomes opaque and a poloidal turning point exists. 
Wave propagation outwards, however, is possible. As the local eigenfrequencies of toroidal and poloidal modes are different any outward propagating large- $m$ poloidal wave may couple to a toroidal oscillation. Poloidal perturbations are related to toroidal transverse currents carrying the wave. As the azimuthal wave number $m$ is large this toroidal current changes sign rapidly also in azimuthal direction. Current continuity requires the transverse divergence of this current to be closed via field-aligned and poloidal currents. This causes coupling between the primary poloidal magnetic field perturbation and a secondary toroidal magnetic field oscillation. If the local eigenfrequencies of the poloidal and toroidal oscillations match a local toroidal field line resonance occurs. Wave propagation is thus restricted to a region bounded by the poloidal turning point and the toroidal resonance point. This region thus defines a magnetospheric resonator or wave guide (Leonovich and Mazur, 1995).

This toroidal resonance is the true equivalent to the classical field line resonance first described by Tamao (1965), Chen and Hasegawa (1974), and Southwood (1974). In case of the classical field line resonance the primary, low$m$ poloidal wave is generated at the magnetopause, while the primary, large- $m$ wave of the magnetospheric resonator field line resonance is generated within the resonator region.

The above discussion indicates that the radial structure of large- $m$ poloidal waves in the magnetospheric resonator will be more complex than that one of a classical field line resonance. A polarization change may happen between the poloidal inner turning point and the outer toroidal resonance. The spatial structure within the resonator needs to be considered.

Furthermore, the outer boundary of the resonator can be another poloidal turning point, not a toroidal resonance, depending on plasma background properties (e.g. Vetoulis and Chen, 1994, 1996; Leonovich and Mazur, 1995; Denton and Vetoulis, 1998; Klimushkin, 1998a). The existence of two radially arranged turning points suggests that a poloidal wave trapped in this kind of resonator can propagate in both directions, inward as well as outward. Detailed studies of the radial variations of the ULF wave field in magnetospheric resonator regions are thus desirable and will allow a deeper insight into the plasma physical processes there.

With the present study we intend to initiate a series of studies on poloidal ULF waves in the outer region of the plasmasphere/inner region of the magnetosphere. In this first report we shall demonstrate the complex spatio-temporal structure of wave fields as seen by the four CLUSTER spacecraft, introduce a new way of representing the observed wave field, and use the theoretical framework of Leonovich and Mazur (1990, 1993, 1995) and Klimushkin (1998a) to model the observed wave fields. Fluxgate magnetometer (FGM) measurements (Balogh et al., 2001) and electric field observations of the EFW instrument (Gustafsson et al., 2001) onboard CLUSTER are analyzed.

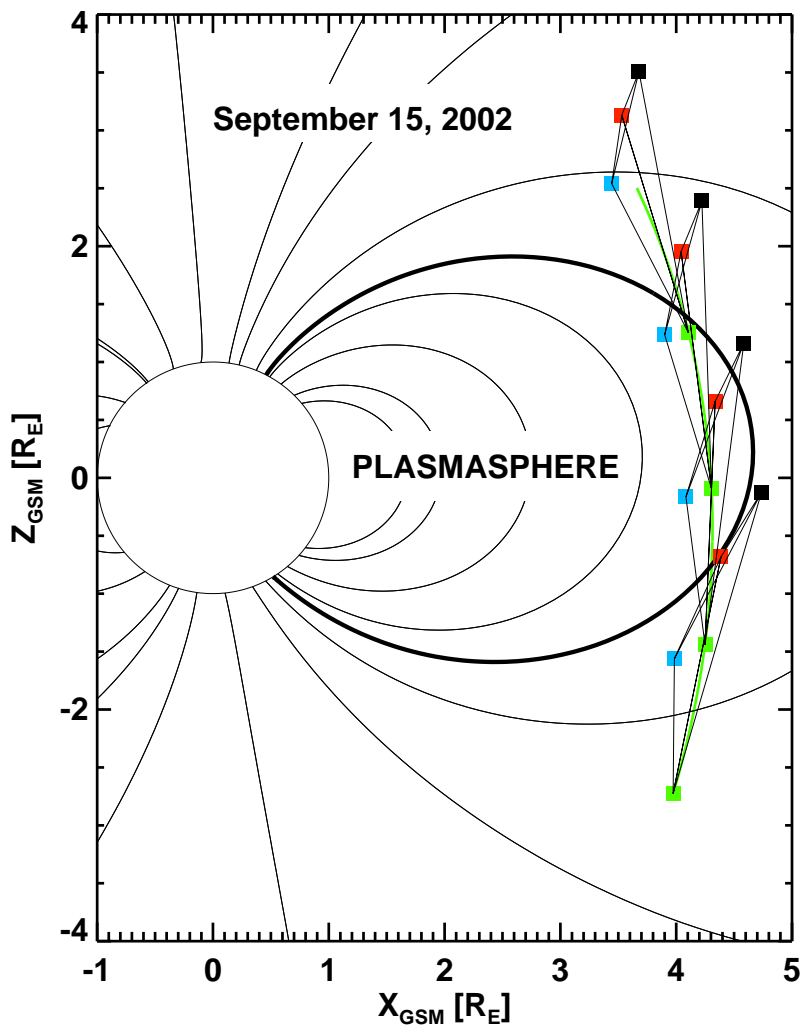

Fig. 1. The CLUSTER orbits in the dayside magnetosphere on 15 September 2002, 06:00, 07:00, 08:00 and 09:00 UT. The satellite move from south to north. The thick black line represents the plasmapause found at $L=4.7$.

\section{Observations}

A poloidal wave event has been detected onboard the CLUSTER spacecraft on 15 September 2002 between 06:50 and 08:00 UT. The CLUSTER orbits during this time interval are displayed in Fig. 1. Field lines have been calculated using the Tsyganenko magnetospheric magnetic field model (Tsyganenko, 1995; Tsyganenko and Stern, 1996). The spacecraft move from the southern dayside magnetosphere into the northern part of the magnetosphere with a velocity of $v_{s c} \approx 2 \mathrm{~km} / \mathrm{s}$. The spacecraft form a large scale tetrahedron with distances between $3000 \mathrm{~km}$ and $16000 \mathrm{~km}$. This configuration provides for an opportunity to investigate ULF pulsations simultaneously observed on different field lines by applying a specific analysis method described in detail further down.

Spatial and temporal parameters of poloidal oscillations are controlled by the local background conditions, e.g. plasma mass density and temperature as well as magnetic field strength and curvature. An accurate interpretation of our observations requires the knowledge of the magnetospheric region where the CLUSTER satellites are located. As the perigee of the spacecraft was rather low they most probably 


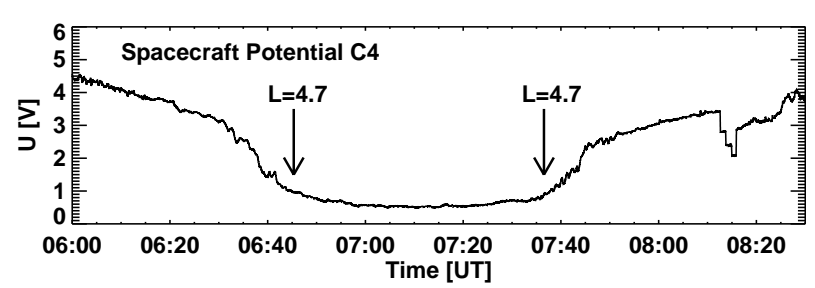

Fig. 2. Spacecraft potential $U$ detected by Cluster C4. The arrows mark the times when spacecraft $\mathrm{C} 4$ crosses the assumed plasmapause position at $L=4.7$ (06:45 and 07:37 UT).

entered the terrestrial plasmasphere and traversed the magnetospheric resonator region discussed in the introduction.

To confirm this the position of the plasmapause is determined using the dynamical simulations of plasmapause formation developed by Pierrard and Lemaire (2004) and Pierrard and Cabrera (2005). This simulation uses the magnetospheric electric field model E5D determined from dynamical proton and electron spectra measured on board the geostationary satellites ATS5 and 6 (McIlwain, 1986). As the electric field model depends only on the $K_{p}$ index the simulation of the plasmapause formation is fully determined by this activity index.

For the given time interval we determine a radial distance of the plasmapause in the magnetic equatorial plane of $4.7 R_{E}$. The field line with the corresponding vertex is assumed as the plasmapause out of the equatorial plane and is indicated by the thick black line in Fig. 1. The assumed plasmapause location at $L=4.7$ is confirmed by the spacecraft potential detected with the EFW instrument on board spacecraft C4 (Fig. 2). A decreased spacecraft potential indicates a higher electron density (Gustafsson et al., 2001; Pedersen et al., 2001). During the time interval analyzed here the solar wind conditions were quiet and stable which justifies the assumption of a constant position of the plasmapause. Except for one all satellites crossed the plasmapause in the Northern and Southern magnetic Hemisphere and thus moved into the dayside plasmasphere. Direct measurements of the electron density as well as ion density and plasma composition by the WHISPER (Décréau et al., 2001) and CIS (Rème et al., 2001) instruments, respectively, onboard CLUSTER do not provide reliable observations for the time period of interest.

Observations used are $4 \mathrm{~s}$ averaged magnetic and electric field measurements. The data are transformed into a MeanField-Aligned (MFA) coordinate system $\left(\boldsymbol{e}_{r}, \boldsymbol{e}_{\phi}, \boldsymbol{e}_{\|}\right)$, where $\boldsymbol{e}_{\|}$denotes the unit vector in the direction of the background magnetic field, $\boldsymbol{e}_{\phi}$ the unit vector perpendicular to the plane spanned by $\boldsymbol{e}_{\|}$and the spacecraft position vector $\boldsymbol{r}_{s c}$. The unit vector $\boldsymbol{e}_{r}$ completes the right hand $\left(\boldsymbol{e}_{r}, \boldsymbol{e}_{\phi}, \boldsymbol{e}_{\|}\right)$triad. The coordinate system used is thus defined as follows:

$\boldsymbol{e}_{r}=\boldsymbol{e}_{\phi} \times \boldsymbol{e}_{\|}$

$$
\begin{aligned}
\boldsymbol{e}_{\phi} & =\frac{\langle\boldsymbol{B}\rangle \times \boldsymbol{r}_{s c}}{\left|\langle\boldsymbol{B}\rangle \times \boldsymbol{r}_{s c}\right|} \\
\boldsymbol{e}_{\|} & =\frac{\langle\boldsymbol{B}\rangle}{|\boldsymbol{B}|} .
\end{aligned}
$$

The mean magnetic field $\langle\boldsymbol{B}\rangle$ is defined as a $512 \mathrm{~s}$ running average magnetic field vector. In the MFA-system $\left(b_{r}, b_{\phi}, b_{\|}\right)$ denote the magnetic disturbance field vectors. As we are only interested in field perturbations the mean value of the fieldaligned component has been subtracted. The MFA-system allows to inspect also the polarization of the analyzed oscillations with $\left(b_{r}, b_{\phi}, b_{\|}\right)$approximately describing poloidal, toroidal, and field-aligned components, respectively.

To describe the electric field perturbations we furthermore assume that $\boldsymbol{E} \times \boldsymbol{B}=0$, i.e. no field-aligned electric field exists. This allows to determine the third electric field component from the two spacecraft spin-plane electric field components provided by the EFW instrument onboard CLUSTER. The vector $\left(E_{r}, E_{\phi}, 0\right)$ denotes the electric field in the MFAsystem.

Observations from all four CLUSTER spacecraft for the time interval studied here are shown in Fig. 3, where the three upper panels display the magnetic field measurements and the two bottom panels the electric field measurements. To discriminate between the four spacecraft they are denoted as $\mathrm{C} 1, \mathrm{C} 2, \mathrm{C} 3$, and $\mathrm{C} 4$, respectively. The pulsation event is most clearly identified in records of spacecraft $\mathrm{C} 3$ and $\mathrm{C} 4$ with an onset time shift of about $30 \mathrm{~min}$ (Fig. 3). The other two spacecraft only detect minor field perturbations.

The frequency of the observed signal is in the range of a Pc4 pulsation, $f \approx 16 \mathrm{mHz}$, observed in all components of the magnetic and electric field. The pulsation at spacecraft $\mathrm{C} 3$ is detected between 07:20 UT and 08:00 UT with transverse electric and magnetic field variations dominating. The amplitudes of $b_{\phi}$ and $b_{r}$ are modulated exhibiting a maximum of about $4 \mathrm{nT}$ in $b_{r}$ and nearly constant field after 07:40 UT. The compressible component $b_{\|}$only shows a small amplitude fluctuation of the order of $1 \mathrm{nT}$. The electric field oscillates with a maximum amplitude of about $2 \mathrm{mV} / \mathrm{m}$ in both components and is almost zero between 07:37 UT and 07:48 UT.

At spacecraft $\mathrm{C} 4$ the oscillation is seen 30 min earlier than at $\mathrm{C} 3$ and ceases at about 07:40 UT. Similar to the observation in $\mathrm{C} 3$ the pulsation is observable in every component of $\boldsymbol{b}$ and $\boldsymbol{E}$. At C4 the radial amplitude $b_{r}$ is twice as large as the azimuthal amplitude $b_{\phi}$ and exhibits a peculiar two-wave packet modulation. The electric field oscillates again with an amplitude of $2 \mathrm{mV} / \mathrm{m}$ and is zero between 07:05 UT and 07:18 UT, whereas the amplitude increases in both components after 07:30 UT up to $5 \mathrm{mV} / \mathrm{m}$ in $E_{\phi}$ and $10 \mathrm{mV} / \mathrm{m}$ in $E_{r}$.

Further information about the observed signal is provided by the Poynting vector $\boldsymbol{S}=\frac{1}{\mu_{0}} \boldsymbol{E} \times \boldsymbol{b}$ and the time-average energy flux

$\langle\boldsymbol{S}\rangle=\frac{1}{T} \int_{0}^{T} \boldsymbol{S} d t$, 


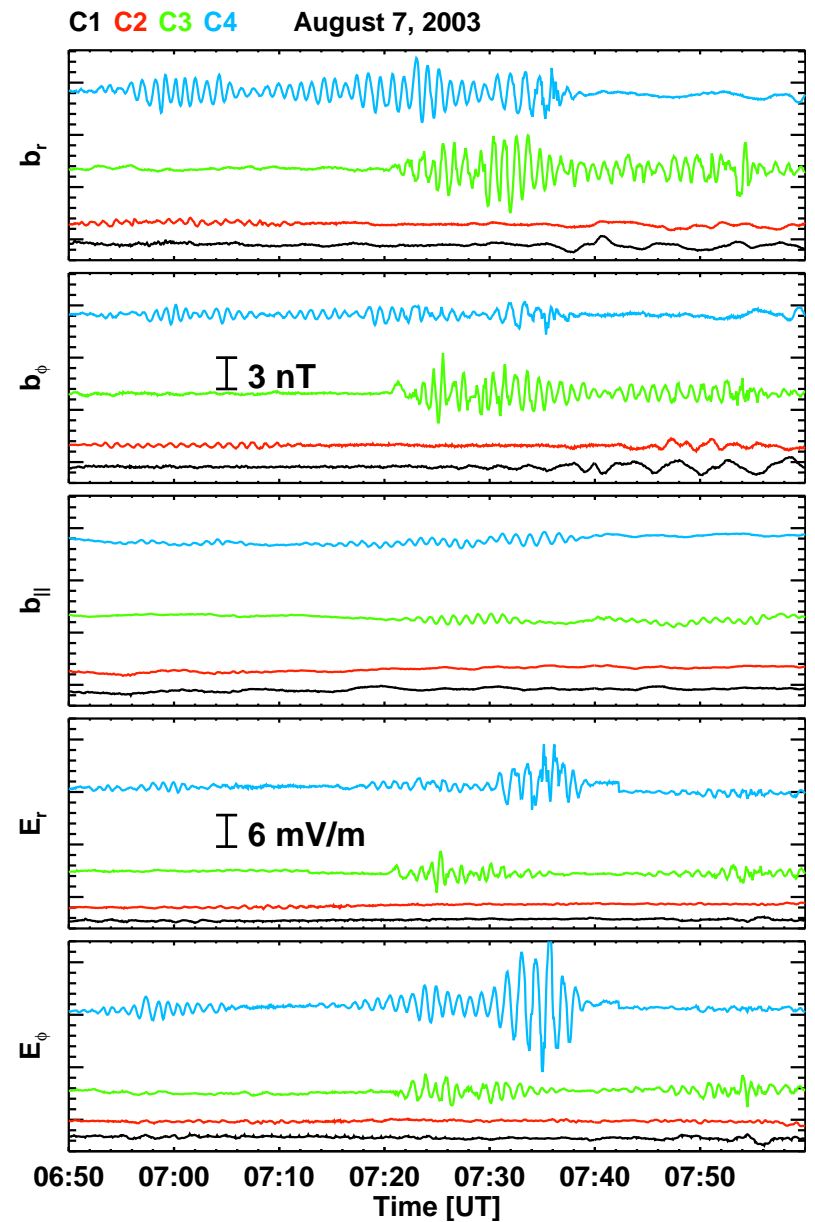

Fig. 3. FGM and EFW measurements for the four Cluster spacecraft (C1: black, $\mathrm{C} 2$ : red, $\mathrm{C} 3$, green, $\mathrm{C} 4$ : blue), transformed into a Mean-Field-Aligned coordinate system. The bars denotes amplitudes of magnetic and electric field, respectively.

where $\mathrm{T}$ is the wave period (Fig. 4), in our case $T=63 \mathrm{~s}$. A standing wave is indicated by $\langle\boldsymbol{S}\rangle=0$ and oscillating Poynting vector $\boldsymbol{S}$ (Chi and Russell, 1998). This is especially observed until 07:20 UT in spacecraft C4. After that time the character of the energy flux changes with both transverse components now indicating a non-zero energy flux transverse to the ambient magnetic field. In particular, the large negative radial component $S_{r}$ corresponds to an inward directed energy transport indicating the presence of a propagating wave. The wave as observed by spacecraft $\mathrm{C} 3$ exhibits small transverse energy flux variations $\left\langle S_{r}\right\rangle$ and $\left\langle S_{\phi}\right\rangle$. The field parallel energy flux is non-zero between 07:20 and 07:34 UT, but the oscillating component of $S_{\|}$is dominant. A non-zero component $\left\langle S_{\|}\right\rangle$is expected for a standing poloidal wave in case of asymmetric ionospheric conductances at the northern and southern footprint of the oscillating field lines (Ozeke et al., 2005). We conclude that at this time interval an almost standing field line oscillation is observed by spacecraft $\mathrm{C} 3$ as well as spacecraft $\mathrm{C} 4$ between 06:50 and 07:20 UT.

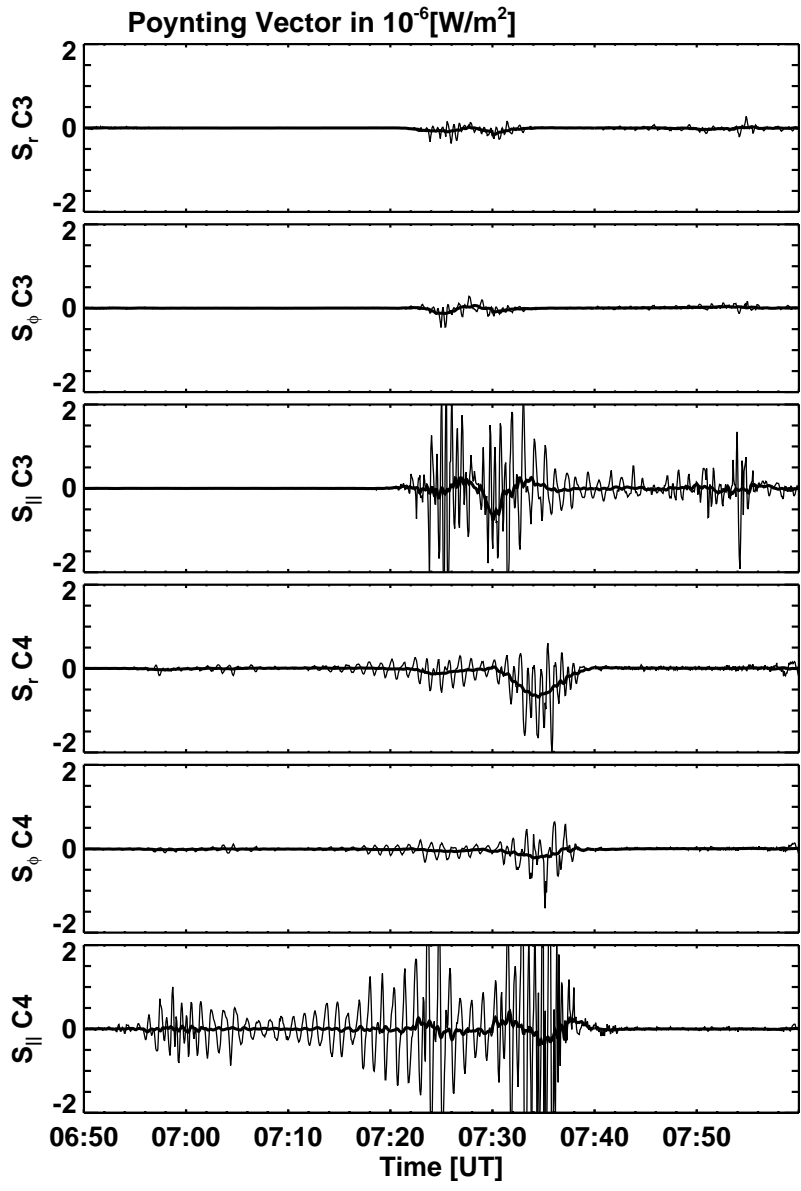

Fig. 4. Components of the Poynting vector $S$ observed by spacecraft $\mathrm{C} 3$ and $\mathrm{C} 4$. The thick line represents the time-average energy flux.

\section{Analysis and interpretation}

\subsection{Phenomenology of the wave activity}

The main aim of the present study is to investigate spatial and temporal structures of poloidal Alfvén waves. In principle, this requires a 4-D-representation of the measurements made. To approach this requirement we introduce a special coordinate system, the L-D-M coordinate system, schematically shown in Fig. 5. The coordinate L resembles the McIlwain Parameter (McIlwain, 1966) and describes the distance of the vertex of a specific field line with respect to the center of the Earth in units of $R_{E}$. To identify the field line for a given point in space the Tsyganenko 96 model (Tsyganenko, 1995; Tsyganenko and Stern, 1996) is used.

The second coordinate $\mathrm{D}$ describes the position of the spacecraft on the specified field line and is defined as the distance between the spacecraft and the field line vertex along the field line. Positive D values correspond to a spacecraft location in the Northern magnetic Hemisphere. The third 


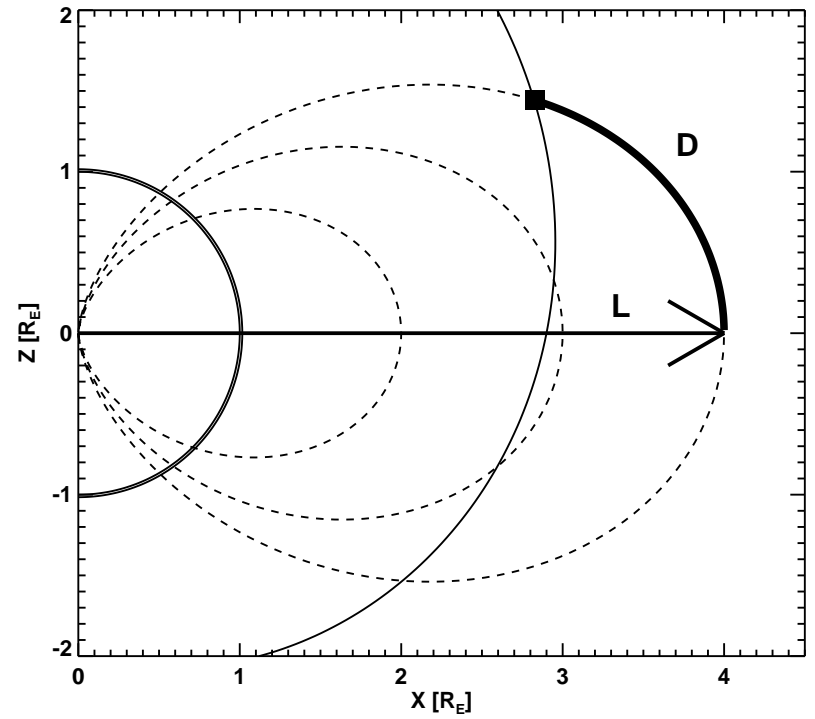

Fig. 5. Schematic illustrating the L-D-M coordinate system. The spacecraft positions are marked by rectangles, the dotted lines represent magnetic field lines.

coordinate, M, describes the magnetic local time (MLT) at which the measurements are taken.

The orbital coordinates of the different spacecraft are transformed into this L-D-M coordinate system, fluctuations of the electromagnetic field are still represented using the Mean-Field-Aligned coordinate system. At first we concentrate on the actual observations of $b_{r}$ and $\left\langle S_{r}\right\rangle$ in the L-D plane as seen in the upper panels of Fig. 6. Effects of minor changes in the M coordinate (lowest panel of Fig. 6) are discussed later. For the representation of $b_{r}$ an analytic signal or Carson-Gabor representation (e.g. Glassmeier, 1980; Glassmeier and Motschmann, 1995) is used, which allows to determine instantaneous amplitude and phase of the given time series. As the ULF signals to be analyzed here are rather regular the analytic signal representation is a very suitable tool. The thickness of the lines denoting the spacecraft positions in the first panel of Fig. 6 represents the instantaneous amplitude or signal envelope of the dominant radial component of the magnetic field oscillation. The thickness of the lines in the second panel of Fig. 6 is related to the radial component of the time averaged energy flux $\left\langle S_{r}\right\rangle$. The vertical black line in Fig. 6 represents the plasmapause position, previously also given in Fig. 1. Wave activity occurs preferentially within the plasmasphere.

In the present case study each spacecraft covers different ranges of $\mathrm{L}$-values. The magnetic equator $(\mathrm{D}=0)$ is crossed by spacecraft $\mathrm{C} 4$ at the field line with its vertex at $L=4.1, \mathrm{C} 3$ at $L=4.35, \mathrm{C} 2$ at $L=4.45$, and $\mathrm{C} 1$ at 4.77 , that is the four spacecraft cover a radial extent of about $0.67 R_{E}$, a value comparable to earlier estimates of the radial extent of magnetospheric pulsations. Therefore, the configuration is suitable to analyze the poloidal oscillation covered spatial region.
Information about the time at which a spacecraft crossed a specific field line is of particular interest. Spacecraft positions at five selected times, labeled by roman numbers, are marked by squares in Fig. 6 . These times have been selected as they correspond to those times spacecraft $\mathrm{C} 4$ and $\mathrm{C} 3$ cross the $\mathrm{L}=4.22$ and $\mathrm{L}=4.4$ field lines in the Southern and Northern Hemisphere and the time spacecraft $\mathrm{C} 4$ detects the maximum, radially directed energy flux.

At time I spacecraft $\mathrm{C} 4$ observed an amplitude maximum at $L=4.22$; the wave amplitude decreased while spacecraft $\mathrm{C} 4$ moved to lower $\mathrm{L}$-values and increased after it crossed the magnetic equator $D=0 R_{E}$. Spacecraft $\mathrm{C} 1$ and C2 were located far away from this field line at $L>4.45$ and did not detect pulsations as seen in the magnetic and electric field measurements (Fig. 3). We conclude that at time I the radial spatial extent of the pulsation event was too small to be observed by $\mathrm{C} 1$ and $\mathrm{C} 2$. Afterwards both spacecraft move further apart from the region of interest and in the following we concentrate on the observations of spacecraft $\mathrm{C} 3$ and $\mathrm{C} 4$. C3 was still too far away from the region of interest to detect any ULF signal at time I.

At time II another amplitude maximum was observed by $\mathrm{C} 4$ at the same field line $L=4.22$ as at time I. This clearly indicates the existence of a radially localized wave structure along this field line oscillating for at least $24 \mathrm{~min}$. At time II a pulsation was also detected by spacecraft $\mathrm{C} 3$, but at $L=4.5$ indicating a more complex radial structure of the wave field observed by the two satellites.

At time III C3 and C4 were located at the same field line shell $L=4.4$, but at slightly different M values. Approaching this field line shell $\mathrm{C} 3$ saw an increasing amplitude, indicating a localized wave packet. At the same time $\mathrm{C} 4$, moving towards the plasmapause only detected a minor change of the field amplitude. This difference in the amplitude behavior might be due to rapid azimuthal variations of the wave field.

Assuming an azimuthal variation $b, E \propto \exp (\operatorname{im} \phi)$ the azimuthal wave number $m$ can be determined from the measured phase difference, $\Delta \psi$, between radial magnetic field variations seen at C3 and C4 (e.g. Eriksson et al., 2005a):

$m=\frac{\Delta \psi}{\Delta \phi}$.

Here $\Delta \phi$ is the azimuthal distance between $\mathrm{C} 3$ and $\mathrm{C} 4$; at time III $\Delta \phi=3.18^{\circ}$. Comparing time series of both spacecraft around time III the phase difference $\Delta \psi$ is estimated. The time difference $\Delta t$ between minima and maxima, respectively, is about $20 \mathrm{~s}$ (Fig. 7), which corresponds to $\Delta \psi \approx 100^{\circ}$; thus $m \approx 30$. Such large azimuthal wave numbers are expected for poloidal oscillations (e.g. Radoski, 1967) and observed by Eriksson et al. (2005b) recently.

After time III spacecraft $\mathrm{C} 4$ recorded regular fluctuations for about $10 \mathrm{~min}$ between $L=4.4 R_{E}$ and $L=4.6 R_{E}$; these were not observed at any of the other spacecraft as they were 


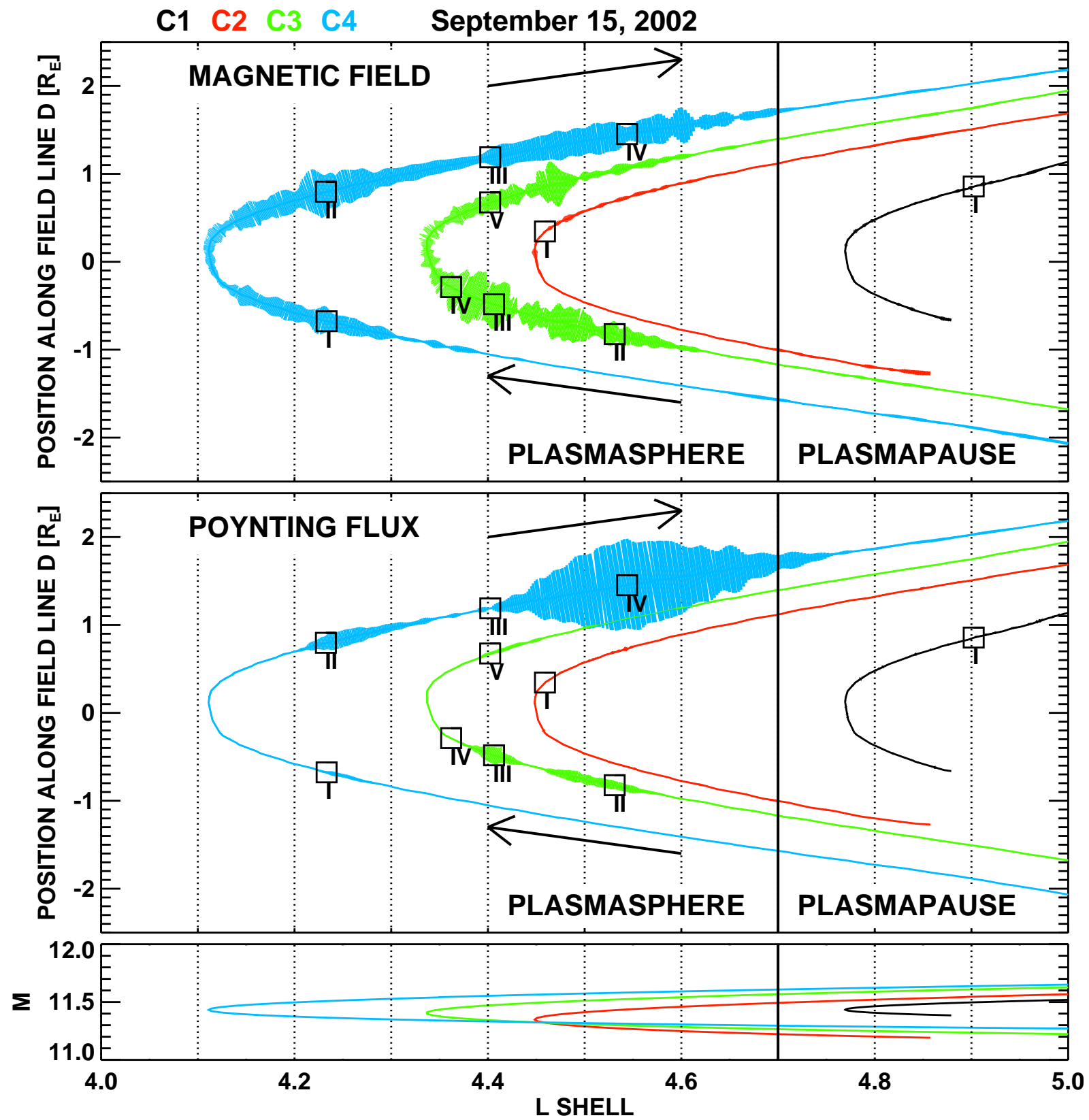

Fig. 6. Positions of the CLUSTER spacecraft in the L-D coordinate system at different times, where the squares mark the positions of each spacecraft. Time I corresponds to 06:58:38 UT, time II to 07:23:42 UT, time III to 07:30:22 UT, time IV to 07:34:22 UT and time V to 07:50:06 UT. The arrows show the flight direction of the CLUSTER satellites. The thickness of the lines are related to the amplitude of the radial oscillation of the magnetic field $b_{r}$ (upper panel) and the radial component of the time averaged Poynting vector $\left\langle S_{r}\right\rangle$ (second panel). The bottom panel shows the orbit in the L-M plane, where M corresponds to the magnetic local time MLT.

located at other field lines. This period of regular oscillations ceased when $\mathrm{C} 4$ reached the plasmapause region.

Between time I and time III the time averaged Poynting vector $\langle\boldsymbol{S}\rangle$ is close to zero indicating the existence of a standing wave field structure. Afterwards spacecraft $\mathrm{C} 4$ observes an increasing radial energy flux with its maximum at time IV located at $L=4.55$ and $D=1.4 R_{E}$. At the same time spacecraft C3 observes $\left\langle S_{r}\right\rangle=0$ at $L=4.36$ and $D=-0.3 R_{E}$. Spacecraft $\mathrm{C} 4$ seems to transit a wave propagating in a defined region as no energy flux is observed by spacecraft $\mathrm{C} 3$. One can speculate that a wave source is located near the observed maximum of the energy flux. The nearby plasmapause at $L=4.7$ acts as a very good wave reflector for the excited wave (e.g. Lee, 1996) which can thus only propagate 


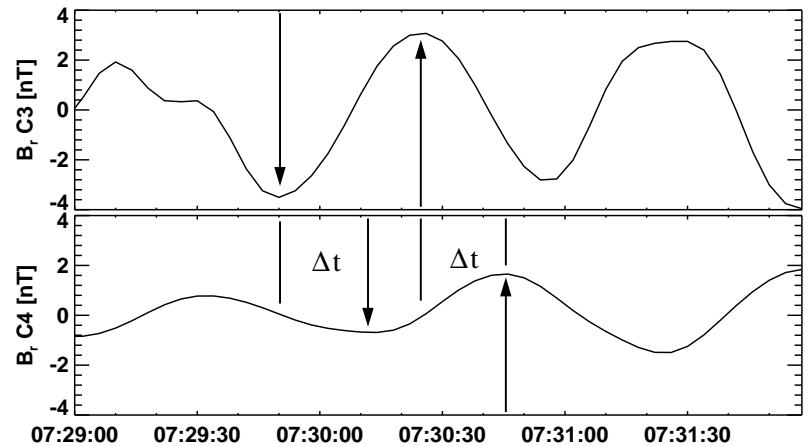

Fig. 7. Time series of $b_{r}$ for spacecraft C3 (top) and C4 (bottom) between 07:29 and 07:32 UT, where both satellites cross the same L-shell at different azimuthal positions $\mathrm{M}$.

towards lower L shells; this is a probable explanation for the observed radially inward directed energy flux.

At time $\mathrm{V}$ spacecraft $\mathrm{C} 3$ again crossed the $\mathrm{L}=4.4$ field line. However, the amplitude was smaller than during the southern crossing, which indicates a temporal variation of the pulsation activity rather than a spatial one. All other spacecraft had already left the activity region at this time.

\subsection{Wave frequency}

Phenomenological characteristics of the waves observed in the outer region of the plasmasphere are quite complex as discussed above. The wave frequency, however, is rather stable at $f_{\mathrm{obs}}=16 \mathrm{mHz}$ during the time spacecraft $\mathrm{C} 3$ and $\mathrm{C} 4$ traversed this region, as seen in the dynamic spectra of the radial magnetic field component $b_{r}$ (Fig. 8). Due to the radial spacecraft motion a frequency constant in time is equivalent to a uniform frequency with respect to magnetic L shells, which has been previously observed and discussed for poloidal wave events in outer magnetospheric regions $(L>6)$ by Denton et al. (2003). Contrary to that the frequency of toroidal waves is expected to have a $L$ dependence and consequently the radial structure of the here observed poloidal wave event cannot be explained by the field line resonance mechanism. Details of the radial structure of poloidal waves can be gained by comparing the observed frequency with the radial profile of theoretically expected poloidal and toroidal eigenfrequencies (e.g. Denton and Vetoulis, 1998; Klimushkin, 1998b).

Theoretically expected toroidal eigenfrequencies $\omega_{T}$ and eigenperiods $\mathrm{T}_{\mathrm{A}}$ of magnetospheric field lines can be estimated using the time of flight method (Warner and Orr, 1979):

$\omega_{T}=\frac{2 \pi N}{T_{A}} ; \quad T_{A}=2 \int_{S}^{N} \frac{d s}{v_{A}}$.

Here $\mathrm{N}$ is the harmonic number of the field line standing oscillation, $d s$ is an increment of length along the field line

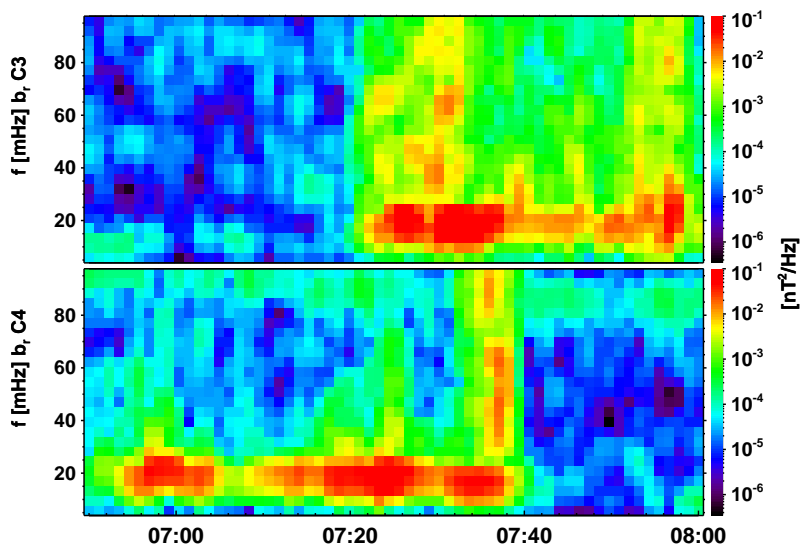

Fig. 8. Dynamic spectra of the radial magnetic field components $b_{r}$ of the spacecraft C3 (top) and C4 (bottom) observations.

and the integration limits are the northern and southern ionosphere. The Alfvén velocity $v_{A}(s)$ along the field line is given by $v_{A}(s)=B(s) / \sqrt{\mu_{0} \rho(s)}$.

Poloidal eigenfrequencies $\omega_{P}$ can be determined in the WKB approximation following Klimushkin (1998a) and Klimushkin (1998b):

$\omega_{P}=\omega_{T}-\omega_{\text {geom }}+\omega_{\beta}$.

Here $\omega_{\text {geom }}$ describes the influence by the field line curvature of a field line and is given by

$\omega_{\text {geom }}=\frac{1}{2 \pi N} \int_{S}^{N} v_{A} \frac{\partial^{2}}{\partial s^{2}}(\ln h) d s$,

where $h$ is a geometric factor depending on the coordinate system used (for details see Klimushkin, 1998a). The term $\omega_{\beta}$ takes into account the influence of finite but small values of the plasma $\beta$ and resulting equilibrium currents $\boldsymbol{J}$ perpendicular to the magnetic field in the equatorial plane, e.g. magnetospheric ring currents:

$\omega_{\beta}=\frac{1}{2 \pi N} \int_{S}^{N} \frac{2 v_{A}}{R}\left(\frac{\mu_{0} J}{B}+\frac{2 v_{M S}^{2}}{R v_{A}^{2}}\right) d s$.

Here $R$ is the field line curvature radius. The slow magnetosonic velocity $v_{M S}$ is given by $v_{M S}=v_{S} v_{A}\left(v_{S}^{2}+v_{A}^{2}\right)^{-1 / 2}$, where $v_{S}$ denotes the sound velocity. For simplicity dipole field lines are assumed to calculate the radius of curvature $R$ and the geometric factor $h$, an assumption well justified for plasmaspheric field lines. The coordinate system of Eq. (13) follows the definiton in Klimushkin (1998a). Here positive values of $J$ correspond to currents directed eastward.

Using Eqs. (10-13) the radial variation of the local poloidal eigenfrequencies $\omega_{P}$ in the outer plasmaspere can be determined using suitable models of the magnetic field, the plasma mass density distribution and the magnetospheric ring current strength. 

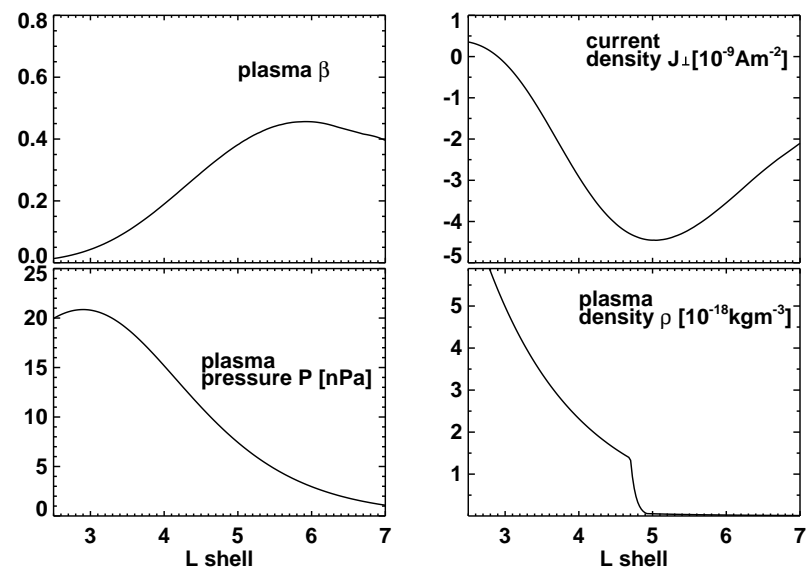

Fig. 9. Profiles of plasma properties. Plasma pressure and plasma $\beta$ are assumed for quiet geomagnetic activity (Lui and Hamilton, 1992). The current density is obtained from the Tsyganenko ' 96 model. Positive values of $J_{\perp}$ correspond to a current directed eastward. The plasma density is calculated from modeling the electron number density (Carpenter and Anderson, 1992).

The latter can be determined using the Tsyganenko model of (Tsyganenko and Stern, 1996; Tsyganenko and Peredo, 1994) for given parameters such as $D_{s t}=-19 \mathrm{nT}$ and the dynamic pressure of the solar wind $p_{\mathrm{dyn}}=0.85 \mathrm{nPa}$. We assume typical distributions for plasma $\beta$ and isotropic plasma pressure $\left(P_{\perp}=P_{\|}\right)$as described e.g. by Lui and Hamilton (1992). To proof consistency with the Tsyganenko model the radial profile of the current density $J$ is also derived from the given plasma pressure distribution by (Lui et al., 1987)

$J_{\perp}=-\frac{1}{B} \frac{\partial P}{\partial L}$.

Here positive $J_{\perp}$ denotes westward current. We found good agreement between the current derived from Eq. (14) and the ring current of the Tsyganenko model. Profiles of $\beta, P$ and $J_{\perp}$ are shown in Fig. 9 .

The Tsyganenko 96 model (Tsyganenko and Stern, 1996) is used to calculate the magnitude of the magnetic field $B(s)$ for a given field line. The plasma number density along the field line is assumed as a power law (Cummings et al., 1969 ) with an exponent $\alpha=1$ typical for the plasmasphere (e.g. Goldstein et al., 2001; Denton et al., 2004):

$n(s)=n_{e q}\left(\frac{L R_{E}}{r}\right)^{\alpha}$.

Here $r$ is the geocentric distance to a point on the field line and the number density in the equatorial plane $n_{e q}$ can be obtained from the model of Carpenter and Anderson (1992). Influences on $n_{e q}$ due to complex features of the plasmapause formation such as plasma plumes or shoulders (e.g. Pierrard and Lemaire, 2004; Goldstein, 2006) are not predicted by the

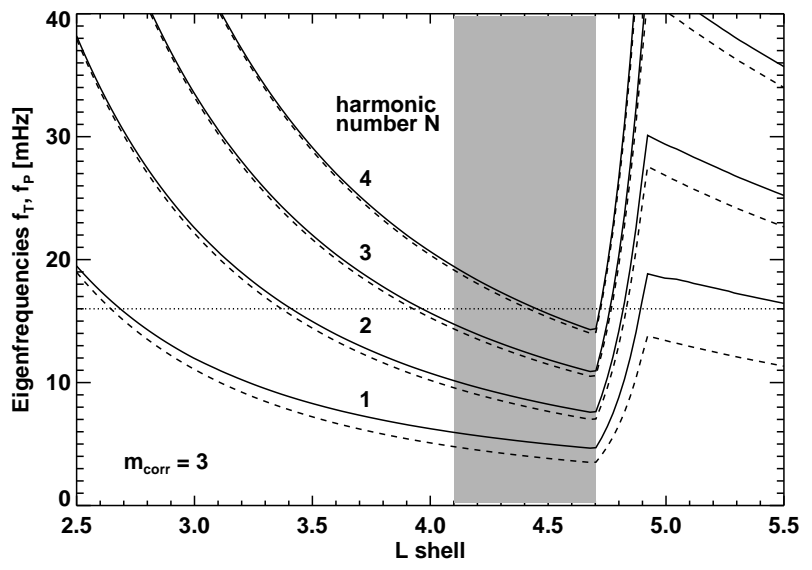

Fig. 10. Profiles of the poloidal eigenfrequencies $f_{P}$ (solid line) and toroidal eigenfrequencies $f_{T}$ (dashed line) of magnetospheric field lines calculated for harmonic numbers $N=1, \ldots, 4$ and the mass correction factor $m_{\text {corr }}=3$. The dotted line marks the observed frequency $f=16 \mathrm{mHz}$. The grey shaded background marks L-shells crossed by spacecraft $\mathrm{C} 3$ and $\mathrm{C} 4$ during the analyzed time interval.

applied simulation of the plasmapause formation, additionally approved by the observed spacecraft potential (Fig. 2). The plasma of the plasmasphere is composed of hydrogen and heavier ions such as helium and oxygen. This requires a mass correction factor $m_{\text {corr }}$ to calculate the plasma mass density $\rho(s)=n(s) m_{\text {corr }} m_{p}$ accurately, where $m_{p}$ is the proton mass. A typical plasmaspheric plasma is composed of $55 \% \mathrm{H}^{+}, 40 \% \mathrm{He}^{+}$and $5 \% \mathrm{O}^{+}$(Berube et al., 2005) leading to a mass correction of $m_{\text {corr }} \approx 3$.

With these assumptions and model values the eigenfrequencies of the fundamental poloidal and toroidal field line oscillation, $f_{P}=\omega_{P} / 2 \pi$ and $f_{T}=\omega_{T} / 2 \pi$, as well as the frequencies of its harmonics $N=2, \ldots, 4$ have been calculated for $\mathrm{L}$ shells in the range $[2.5,5.5] R_{E}$ (Fig. 10). At L shells $L=[4.1,4.7]$, where spacecraft $\mathrm{C} 3$ and $\mathrm{C} 4$ observe the described pulsation activity, poloidal eigenfrequencies are larger than the toroidal eigenfrequencies; the non zero plasma $\beta$ leads to $\omega_{\beta}>0$. The geometric contribution, $\omega_{\text {geom }}$, is always larger than zero for $\alpha=6$. However, if we assume $\alpha \neq 6$, negative values for $\omega_{\text {geom }}$ are well possible. In the present case $\alpha=1$ is the best guess and we find $\omega_{\text {geom }}<0$ for certain radial distances. This leads to $\omega_{P}>\omega_{T}$ in these regions.

The theoretical framework predicts the existence of a region transparent for poloidal waves near a minimum of $f_{P}$ only if the condition $f_{P}<f_{\text {obs }}<f_{T}$ is satisfied (Klimushkin et al., 2004). In contrast to that we found $f_{T}<f_{P}<f_{\text {obs }}$ in the present case study (Fig. 10). However, the theory used here describes wave fields bounded by surfaces satisfying the condition $f_{\mathrm{obs}}=f_{P}$. These surfaces act as turning points of the radial propagating wave and define a region transparent for poloidal waves. Consequently, we assume that the observed 
pulsation is localized within such a wave transparency region despite the contradiction between observation and theory. A possible reason for this discrepancy is the negative value of $\omega_{\text {geom }}$ due to the assumed exponent $\alpha=1$ in Eq. (15), as discussed above. As the condition $\omega_{\text {geom }}>\omega_{\beta}$ is necessary to obtain a poloidal eigenfrequency smaller than the toroidal (see Eq. 11), we speculate that Eq. (12) is inappropriate in case of $\alpha=1$. Apparently further efforts are necessary to improve the applied WKB approximation of the theoretical framework using the constraint $\alpha=1$.

However, we suggest that Fig. 10 reflects the radial width of the poloidal resonator (e.g. Leonovich and Mazur, 1993). The outer boundary coincides with the plasmapause at $L \approx 4.7$ and the inner boundary depends on the harmonic number $N$. Since spacecraft $\mathrm{C} 4$ observes the pulsation even at $L=4.1$, we exclude the fourth harmonic oscillation, which has the inner resonance surface located at $L \approx 4.2$. The observations described in the L-D coordinate system (Fig. 6) reveal a symmetric amplitude structure relative to the magnetic equator $D=0 R_{E}$ suggesting an oscillation with an odd harmonic number. For the fundamental oscillation $N=1$ the spatial extent of the oscillating structure would be more than $2 R_{E}$. Consequently, we suggest that the observed pulsation is a third harmonic oscillation with a spatial extent of approximately $0.7 R_{E}$.

\subsection{Modeling the spatio-temporal structure}

For further understanding of the observed pulsation event and to reach a deeper insight into its spatio-temporal structure we present a simple model for the spatio-temporal characteristics of the wave which is fitted to the actually observed data for better comparison. The dominant poloidal magnetic field component is given by

$b_{r \text { model }}=b_{0} \cdot A(D, \phi) \cdot B(L) \cdot C(t)$.

Here $A(D, \phi)$ describes the spatial structure along the field line and in the azimuthal direction, $B(L)$ the spatial structure in the radial direction across L-shells, and $C(t)$ the temporal evolution of the wave amplitude. All three amplitude functions are normalized to 1 , so that the maximum amplitude of the modeled signal is given by $b_{0}$.

The model assumes a standing wave along the background magnetic field line:

$A(D, \phi)=\sin \left(k_{\|} D+m \phi\right) \cdot \cos \left(\omega_{P} t\right)$.

This functional form describes odd mode oscillations with $k_{\|}$denoting the field parallel wave number. Wave frequency $\omega_{P}=2 \pi f_{P}$ and azimuthal wave number $m$ are determined using the observations. As mentioned in Sect. 2 the change in the azimuthal spacecraft position $\Delta \phi=\Delta M$ is small, but since we found $m \approx 30$, the phase variation $\Delta(m \phi)$ is not negligible. The parallel wavelength $\lambda_{\|}=2 \pi / k_{\|}$depends on the length of the field line $l$ and the harmonic number $N: \lambda_{\|}=2 l / N$. The lengths of the field lines with vertices between $L=4.1 R_{E}$ and $L=4.7 R_{E}$ are between $l=9.4$ and $l=10.0 R_{E}$. Assuming a third harmonic oscillation as discussed above gives one wavelengths in the range $\lambda_{\|}=[6.3,6.7] R_{E}$ or wave numbers $k_{\|}=[0.94,1.00] R_{E}^{-1}$.

The transverse variation of the wave field is described by multiplying the standing wave (Eq. 17) with an amplitude function $B(L)$. Leonovich and Mazur (1990) have shown that for a poloidal wave resonator the function $B(L)$ can be approximated by the product of a Hermitian polynomial $H_{n}$ of order $\mathrm{n}$ and a Gaussian:

$B(L)=H_{n}(\xi) \exp \left(-\xi^{2} / 2\right)$,

where $\xi=\left(L-L_{R}\right) / \sigma$. Here $L_{R}$ denotes the location of maximum wave amplitude and $\sigma$ describes the radial width of the wave field within the wave guide region, respectively. For a zeroth order Hermitian polynomial $(n=0)$ the radial structure $B(L)$ is just given by a Gaussian as already observed for poloidal pulsations (e.g. Cramm et al., 2000).

Using higher order Hermitian polynomials allows to describe a more complex wave field variation in radial direction as the number of extrema of the polynomial used is equal to $n+1$. Figure 6 exhibits two amplitude maxima at $L=4.22$ and $L=4.40 R_{E}$. The first one at $L=4.22 R_{E}$ is detected twice by spacecraft $\mathrm{C} 4$ when it crosses the corresponding field line below and above the magnetic equator. The second one at $L=4.40 R_{E}$ is detected only by C3. Due to this observation a first order Hermitian polynomial with $n=1$ is used in Eq. (18).

The amplitude maxima on the field line $L=4.22 R_{E}$ are observed at $D=-0.7 R_{E}$ and $D=0.9 R_{E}$, that is almost symmetric with respect to the magnetic equator. Assuming a standing wave, one would expect nearly the same pulsation strength for both crossings. However, the opposite is observed with the northern maximum displaying a somewhat small amplitude value (Fig. 6). This is either due to the symmetry point not coinciding with the magnetic equator or the result of a temporal evolution of the wave field, approximated by a Gaussian function $C(t)$ with its maximum at 07:32 UT corresponding to the observed maximum of the signal. An increasing amplitude can be explained by e.g. drift bounce resonance effects, as described in Klimushkin and Mager (2004). On the other hand, wave dissipation at the ionospheric boundaries leads to a decreasing pulsation amplitude.

The aim of our modeling effort is to characterize the amplitude $b_{0}$, the activity maximum position $L_{R}$ and its width $\sigma$. Varying these model parameters the best correspondence between modeled signal and the actual observations of spacecraft C3 and C4 have been reached for $L_{R}=4.35 R_{E}$, $\sigma=0.1 R_{E}, m=30$, and $b_{0}=6.5 \mathrm{nT}$. A comparison of both signals is displayed in Fig. 11. The correspondence is fairly reasonable with major features of the observed amplitude modulation being explained. However, it is not possible to reproduce the measurements in detail. The first amplitude maximum observed at spacecraft $\mathrm{C} 4$ observation is not fully reproduced by the model such as the amplitude modulation 


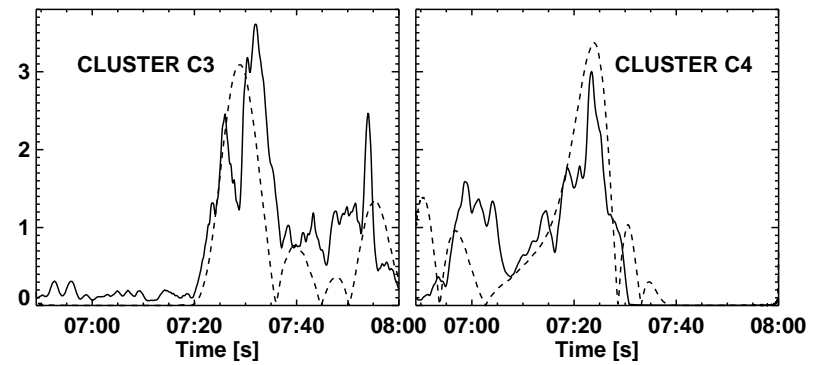

Fig. 11. Comparison between measurement of the $b_{r}$ (solid line) component and the model of the wave field $b_{r, \text { model }}$ (dashed line).

seen in C3 after 07:35 UT. Observations between 07:10 and 07:35 UT are well represented by the modeled signal. Especially the modeled shape of the second amplitude peak in spacecraft $\mathrm{C} 4$ is in a good agreement with the observed amplitude modulation.

Figure 12 shows a comparison between the modeled radial variation of the wave field and the radial variation of the poloidal eigenfrequency. It is remarkable to see that the wave activity region as modeled well coincides with the wave guide region identified as the trough in the poloidal eigenfrequency variation. From the modeled signal we also infer that the total width of the wave guide is about $0.6 R_{E}$, which corresponds to the observed extend of the wave activity region.

It should be noted that the time interval between 07:30 and 07:40 UT of the spacecraft C4 observation is excluded from our modeling efforts as at this time a propagating wave is detected (Figs. 4 and 6) and the assumption of a standing wave is not suitable in this region.

Differences between model and measurements originate from several sources. First, the Hermitian function used to describe the radial structure is based on the assumption of a wave guide symmetric to the point of minimum eigenfrequency. This assumption is not fully consistent with the actual variation of the poloidal eigenfrequency. Second, theoretical studies such as presented by Klimushkin and Mager (2004) assume a time harmonic time variation without any amplitude modulation. A more realistic temporal evolution of the wave field is not incorporated in current theoretical treatments. We think that this oversimplification is the main reason for discrepancies between model and observation. However, similarities found suggest that the theoretical assumptions allow for explaining the observed amplitude modulation, at least after the wave field is fully developed and before it collapsed due to ionospheric dissipation.

\section{Conclusions}

A new representation of magnetic field measurements has been developed which allows a very convenient graphical display of CLUSTER multi-spacecraft observations helping

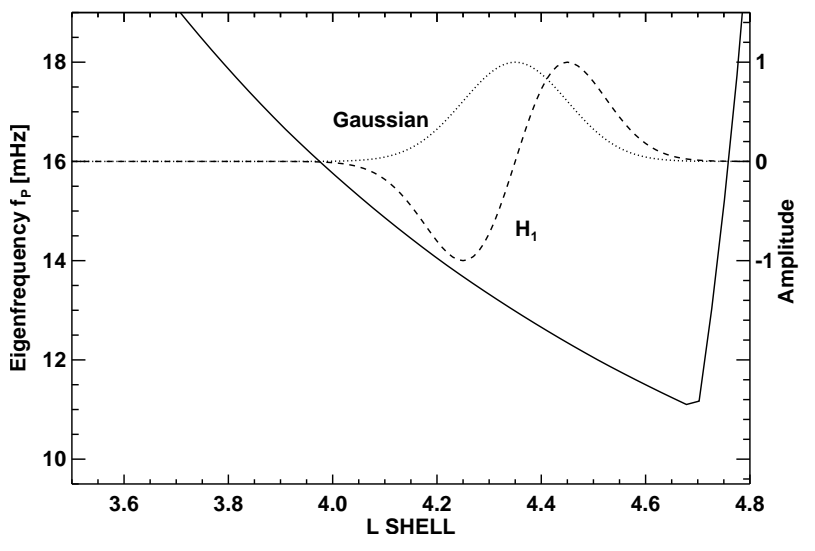

Fig. 12. Comparison between the modeled radial variation and the radial variation of the poloidal eigenfrequency.

to visualize spatial and temporal effects due to spacecraft motion. This new visualization tool has been applied to ULF pulsation observations in the terrestrial dayside plasmasphere and a region of a poloidal standing wave was detected and analyzed.

The field line oscillations analyzed were observed in the time interval 15 September 2002, 06:50-08:00 UT. The large scaled spacecraft configuration during this interval has entailed the possibility to record the pulsation event over a time period of around $70 \mathrm{~min}$ in spacecraft $\mathrm{C} 3$ and $\mathrm{C} 4$. During this time the spacecraft crossed the oscillating field lines twice during their inbound and outbound approaches to the Earth on an almost polar orbit.

Analyzing the phase differences between signals observed by two spacecraft while passing the same $\mathrm{L}$ shell has given us the possibility to estimate the azimuthal wave number; we found $m \approx 30$. Accordingly, a poloidal polarized oscillation with $m \gg 1$ was detected.

To describe temporal and spatial properties of the observed wave field we have used a theoretical framework developed by Leonovich and Mazur $(1990,1993,1995)$ and Klimushkin (1998a). A profile of poloidal eigenfrequencies $f_{P}$ was evaluated based on realistic assumption concerning plasma composition and plasma properties in the region of interest. Comparing with the observed frequency $f_{\mathrm{obs}}=16 \mathrm{mHz}$ has suggested a third harmonic oscillation.

The wave field was found localized near the minimum of $f_{P}$ bounded by turning points $f_{P}=f_{\text {obs }}$ at the plasmapause and within the plasmasphere, respectively. In this region the existence of a localized pulsation is not fully predicted by theory. Hence, further efforts on the theoretical description of plasmaspheric ULF pulsations are necessary.

Modeling spacecraft observations crossing plasmaspheric field lines has suggested a spatial structure and temporal development of the detected wave field. We have found clear indications of the existence of a standing wave at $L=4.22 R_{E}$ 
together with a complex radial structure extended to higher L-shells. The wave event is localized in radial direction. However, this localization is not due to any resonant mode coupling, but thought to be the result of the wave field encountering two poloidal turning points, much as predicted by Klimushkin (1998a). The spatial extent of the wave field area is around $0.6 R_{E}$, which confirms previous observations of plasmaspheric ULF pulsations (Ziesolleck et al., 1993; Menk et al., 1999). The radial structure was described by a specific function as suggested by Leonovich and Mazur (1990). In addition to the standing structure the investigation of the wave Poynting vector has clearly exposed the existence of an inward directed, propagating wave.

Comparing observations with the modeled standing oscillation exposes strengths and weak points of the applied model and its theoretical basis. For the given situation of a solely poloidal polarized field line oscillation a theory is currently unavailable specifying the temporal evolution of such a wave field. Thus, the measured pulsation can only be reproduced in parts by the modeled standing structure.

Our future efforts will concentrate on the performance of further event studies of ULF pulsations localized within plasmasphere and plasmapause. We intend to analyze these events with the method introduced by the present work. In addition to that we aim for the development of a statistical survey of properties of Alfvénic waves in the terrestrial magnetosphere. For these purposes the CLUSTER mission is an appropriate tool providing us with an extensive amount of magnetic and electric field data over a time range of more than five years.

Acknowledgements. This work was financially support by the German Ministerium für Wirtschaft und Technologie and the Deutsches Zentrum für Luft- und Raumfahrt under contract 50OC0103. Part of this work was financially supported by INTAS under contract 05 1000008-7978.

Topical Editor I. A. Daglis thanks S. Buchert and another referee for their help in evaluating this paper.

\section{References}

Balogh, A., Carr, C. M., Acuna, M. H., and Dunlop, M. W.: The Cluster magnetic field investigation: overwiew of in-flight perfomance and initial results, Ann. Geophys., 19, 1207-1217, 2001, http://www.ann-geophys.net/19/1207/2001/.

Berube, D., Moldwin, M. B., Fung, S. F., and Green, J. L.: A plasmaspheric mass density model and constraints on its heavy ion concentration, J. Geophys. Res. (Space Physics), 110, A04212, 1-5, doi:10.1029/2004JA010684, 2005.

Carpenter, D. L. and Anderson, R. R.: An ISEE/Whistler model of equatorial electron density in the magnetosphere, J. Geophys. Res., 97, 1097-1108, 1992.

Chen, L. and Hasegawa, A.: A theory of long-periodic magnetic pulsations, 1. Steady excitation of field line resonances, J. Geophys. Res., 79, 1024-1032, 1974.
Chi, P. J. and Russell, C. T.: Phase skipping and Poynting flux of continuous pulsations, J. Geophys. Res., 103, 29 479-29492, 1998.

Cramm, R., Glassmeier, K. H., Othmer, C., Fornacon, K. H., Auster, H. U., Baumjohann, W., and Georgescu, E.: A case study of a radially polarized Pc4 event observed by the Equator-S satellite, Ann. Geophys., 18, 411-415, 2000, http://www.ann-geophys.net/18/411/2000/.

Cummings, W. D., O'Sullivan, R. J., and Coleman, P. J.: Standing Alfvén Waves in the Magnetosphere, J. Geophys. Res., 74, 778793, 1969.

Décréau, P. M. E., Fergeau, P., Krasnoselskikh, V., Le Guirriec, E., Lévêque, M., Martin, P., Randriamboarison, O., Rauch, J. L., Sené, F. X., Séran, H. C., Trotignon, J. G., Canu, P., Cornilleau, N., de Féraudy, H., Alleyne, H., Yearby, K., Mögensen, P. B., Gustafsson, G., André, M., Gurnett, D. C., Darrouzet, F., Lemaire, J., Harvey, C. C., Travnicek, P., and Whisper Experimenters group: Early results from the Whisper instrument on Cluster: an overview, Ann. Geophys., 19, 1241-1258, 2001, http://www.ann-geophys.net/19/1241/2001/.

Denton, R. E. and Vetoulis, G.: Global poloidal mode, J. Geophys. Res., 103, 6729-6740, doi:10.1029/97JA03594, 1998.

Denton, R. E., Lessard, M. R., and Kistler, L. M.: Radial localization of magnetospheric guided poloidal Pc 4-5 waves, J. Geophys. Res. (Space Physics), 108, SMP 4, 1-10, doi:10.1029/ 2002JA009679, 2003.

Denton, R. E., Menietti, J. D., Goldstein, J., Young, S. L., and Anderson, R. R.: Electron density in the magnetosphere, J. Geophys. Res. (Space Physics), 109, A09215, 1-14, doi:10.1029/ 2003JA010245, 2004.

Engebretson, M., Glassmeier, K.-H., Stellmacher, M., Hughes, W. J., and Lühr, H.: The dependence of high-latitude Pc5 wave power on solar wind velocity and on the phase of highspeed solar wind streams, J. Geophys. Res., 103, 26 271-26284, doi:10.1029/97JA03143, 1997.

Engebretson, M. J. and Cahill, L. J.: Pc5 pulsations observed during the June 1972 geomagnetic storm, J. Geophys. Res., 86, 56195631, doi:10.1029/0JGREA0000860000A7005619000001, 1981.

Engebretson, M. J., Zanetti, L. J., Potemra, T. A., and Acuna, M. H.: Harmonically structured ULF pulsations observed by the AMPTE CCE magnetic field experiment, Geophys. Res. Lett., 13, 905-908, 1986.

Eriksson, P. T. I., Blomberg, L. G., and Glassmeier, K.-H.: Cluster satellite observations of $\mathrm{mHz}$ pulsations in the dayside magnetosphere, Adv. Space Res., 23, 2679-2685, doi:10.1016/j.asr.2005. 04.103, 2005a.

Eriksson, P. T. I., Blomberg, L. G., Walker, A. D. M., and Glassmeier, K.-H.: Poloidal ULF oscillations in the dayside magnetosphere: a Cluster study, Ann. Geophys., 23, 2679-2685, 2005 b.

Escoubet, C. P., Schmidt, R., and Goldstein, M. L.: Cluster - Science and Mission Overview, Space Sci. Rev., 79, 11-32, 1997.

Glassmeier, K. H.: Magnetometer array observations of a giant pulsation event, Journal of Geophysics Zeitschrift Geophysik, 48, 127-138, 1980.

Glassmeier, K. H. and Motschmann, U.: Comments on Time-Series Analysis, ESA SP-371: Proceedings of the Cluster Workshops, Data Analysis Tools and Physical Measurements and MissionOriented Theory, pp. 7-14, 1995. 
Glassmeier, K. H., Volpers, H., and Baumjohann, W.: Ionospheric Joule dissipation as a damping mechanism for high latitude ULF pulsations: Observational evidence, Planet. Space Sci., 32, 1463-1466, 1984.

Goldstein, J.: Plasmasphere Response: Tutorial and Review of Recent Imaging Results, Space Science Reviews, 124, 203-216, doi:10.1007/s11214-006-9105-y, 2006.

Goldstein, J., Denton, R. E., Hudson, M. K., Miftakhova, E. G., Young, S. L., Menietti, J. D., and Gallagher, D. L.: Latitudinal density dependence of magnetic field lines inferred from Polar plasma wave data, J. Geophys. Res., 106, 6195-6202, doi:10. 1029/2000JA000068, 2001.

Gustafsson, G., André, M., Carozzi, T., Eriksson, A. I., Fälthammar, C.-G., Grard, R., Holmgren, G., Holtet, J. A., Ivchenko, N., Karlsson, T., Khotyaintsev, Y., Klimov, S., Laakso, H., Lindqvist, P.-A., Lybekk, B., Marklund, G., Mozer, F., Mursula, K., Pedersen, A., Popielawska, B., Savin, S., Stasiewicz, K., Tanskanen, P., Vaivads, A., and Wahlund, J.-E.: First results of electric field and density observations by Cluster EFW based on initial months of operation, Ann. Geophys., 19, 1219-1240, 2001 ,

http://www.ann-geophys.net/19/1219/2001/.

Hasegawa, A.: Drift Mirror Instability in the Magnetosphere, Phys. Fluids, 12, 2642-2650, 1969.

Kivelson, M. G. and Southwood, D. J.: Coupling of global magnetospheric MHD eigenmodes to field line resonances, J. Geophys. Res., 91, 4345-4351, 1986

Klimushkin, D. and Mager, P.: The spatio-temporal structure of impulse-generated azimuthalsmall-scale Alfvén waves interacting with high-energy charged particles in the magnetosphere, Ann. Geophys., 22, 1053-1060, 2004, http://www.ann-geophys.net/22/1053/2004/.

Klimushkin, D., Mager, P., and Glassmeier, K.: Toroidal and poloidal Alfvén waves with arbitrary azimuthal wavenumbers in a finite pressure plasma in the Earth's magnetosphere, Ann. Geophys., 22, 267-287, 2004, http://www.ann-geophys.net/22/267/2004/.

Klimushkin, D. Y.: Resonators for hydromagnetic waves in the magnetosphere, J. Geophys. Res., 103, 2369-2376, doi:10.1029/ 97JA02193, 1998a.

Klimushkin, D. Y.: Theory of azimuthally small-scale hydromagnetic waves in the axisymmetric magnetosphere with finite plasma pressure, Ann. Geophys., 16, 303-321, 1998b.

Lee, D.-H.: Dynamics of MHD wave propagation in the lowlatitude magnetosphere, J. Geophys. Res., 101, 15371-15386, doi:10.1029/96JA00608, 1996.

Lee, D.-H. and Lysak, R. L.: Effects of azimuthal asymmetry on ULF waves in the dipole magnetosphere, Geophys. Res. Lett., 17, 53-56, 1990.

Leonovich, A. S. and Mazur, V. A.: The spatial structure of poloidal alfven oscillations of an axisymmetric magnetosphere, Planet. Space Sci., 38, 1231-1241, doi:10.1016/0032-0633(90) 90128-D, 1990

Leonovich, A. S. and Mazur, V. A.: A theory of transverse small-scale standing Alfvén waves in an axially symmetric magnetosphere, Planet. Space Sci., 41, 697-717, doi:10.1016/ 0032-0633(93)90055-7, 1993

Leonovich, A. S. and Mazur, V. A.: Magnetospheric resonator for transverse-small-scale standing Alfven waves, Planet. Space
Sci., 43, 881-883, 1995.

Lui, A. T. Y. and Hamilton, D. C.: Radial profiles of quiet time magnetospheric parameters, J. Geophys. Res., 97, 19325-19332, 1992.

Lui, A. T. Y., McEntire, R. W., and Krimigis, S. M.: Evolution of the ring current during two geomagnetic storms, J. Geophys. Res., 92, 7459-7470, 1987.

Mann, I. R. and Wright, A. N.: Finite lifetimes of ideal poloidal Alfvén waves, J. Geophys. Res., 100, 23 677-23 686, doi:10. 1029/95JA02689, 1995.

Mcllwain, C. E.: Magnetic Coordinates, Space Sci. Rev., 5, 585598, 1966

McIlwain, C. E.: A Kp dependent equatorial electric field model, Adv. Space Res., 6, 187-197, doi:10.1016/0273-1177(86) 90331-5, 1986.

Menk, F. W., Orr, D., Clilverd, M. A., Smith, A. J., Waters, C. L., Millng, D. K., and Fraser, B. J.: Monitoring spatial and temporal variations in the dayside plasmasphere using geomagnetic field line resonances, J. Geophys. Res., 104, 19955-19970, 1999.

Mitchell, D. G., Williams, D. J., Engebretson, M. J., Cattell, C. A., and Lundin, R.: Pc 5 pulsations in the outer dawn magnetosphere seen by ISEE 1 and 2, J. Geophys. Res., 95, 967-975, 1990.

Olson, J. V. and Rostoker, G.: Longitudinal phase variations of Pc 4-5 micropulsations, J. Geophys. Res., 83, 2481-2488, 1978.

Ozeke, L. G. and Mann, I. R.: Modeling the properties of high$m$ Alfvén waves driven by the drift-bounce resonance mechanism, J. Geophys. Res., 106, 15 583-15 598, doi:10.1029/ 2000JA000393, 2001.

Ozeke, L. G., Mann, I. R., and Mathews, J. T.: The influence of asymmetric ionospheric Pedersen conductances on the fieldaligned phase variation of guided toroidal and guided poloidal Alfvén waves, J. Geophys. Res. (Space Physics), 110, A08 210 , 1-16, doi:10.1029/2005JA011167, 2005.

Pedersen, A., Décréau, P., Escoubet, C.-P., Gustafsson, G., Laakso, H., Lindqvist, P.-A., Lybekk, B., Masson, A., Mozer, F., and Vaivads, A.: Four-point high time resolution information on electron densities by the electric field experiments (EFW) on Cluster, Ann. Geophys., 19, 1483-1489, 2001, http://www.ann-geophys.net/19/1483/2001/.

Pierrard, V. and Cabrera, J.: Comparisons between EUV/IMAGE observations and numerical simulations of the plasmapause formation, Annales Geophysicae, 23, 2635-2646, 2005.

Pierrard, V. and Lemaire, J. F.: Development of shoulders and plumes in the frame of the interchange instability mechanism for plasmapause formation, Geophys. Res. Lett., 31, 5809-5813, doi:10.1029/2003GL018919, 2004

Radoski, H. R.: Highly asymmetric MHD resonance: The guided poloidal mode, J. Geophys. Res., 72, 4026-4027, 1967.

Rème, H., Aoustin, C., Bosqued, J. M., and Dandouras, I.: First multispacecraft ion measurements in and near the Earth's magnetosphere with the identical Cluster ion spectrometry (CIS) experiment, Ann. Geophys., 19, 1303-1354, 2001, http://www.ann-geophys.net/19/1303/2001/.

Samson, J. C., Jacobs, J. A., and Rostoker, G.: Latitude-Dependenct Characteristic of Long-Period Geopmagnetic Micropulsations, J. Geophys. Res., 76, 3675-3683, 1971.

Singer, H. J.: Multisatellite observations of resonant hydromagnetic waves, Planet. Space Sci., 30, 1209-1218, 1982.

Singer, H. J., Russell, C. T., Kivelson, M. G., Fritz, T. A., and 
Lennartsson, W.: Satellite observations of the spatial extent and structure of Pc 3, 4, 5 pulsations near the magnetospheric equator, Geophys. Res. Lett., 6, 889-892, 1979.

Singer, H. J., Hughes, W. J., and Russell, C. T.: Standing hydromagnetic waves observed by ISEE 1 and 2 - Radial extent and harmonic, J. Geophys. Res., 87, 3519-3529, 1982.

Southwood, D. J.: Some features of field line resonances in the magnetosphere, Planet. Space Sci., 22, 483-491, 1974.

Southwood, D. J. and Kivelson, M. G.: Charged particle behavior in low-frequency geomagnetic pulsations. II - Graphical approach, J. Geophys. Res., 87, 1707-1710, 1982.

Southwood, D. J., Dungey, J. W., and Etherington, R. J.: Bounce resonant interaction between pulsations and trapped particles, Planet. Space Sci., 17, 349-361, 1969.

Tamao, T.: Transmission and coupling resonance of hydrodynamic disturbances in the non-uniform Earth's magnetosphere, Sci. Rep. Tohoku Univ., Ser 5, 17, 1965.

Tsyganenko, N. A.: Modeling the Earth's magnetospheric magnetic field confined within a realistic magnetopause, J. Geophys. Res., 100, 5599-5612,, 1995.
Tsyganenko, N. A. and Peredo, M.: Analytical models of the magnetic field of disk-shaped current sheets, J. Geophys. Res., 99, 199-205, 1994.

Tsyganenko, N. A. and Stern, D. P.: Modeling the global magnetic field of the large-scale Birkeland current systems, J. Geophys. Res., 101, 27 187-27 198, 1996.

Vetoulis, G. and Chen, L.: Global structures of Alfven-ballooning modes in magnetospheric plasmas, Geophys. Res. Lett., 21, 2091-2094, 1994.

Vetoulis, G. and Chen, L.: Kinetic theory of geomagnetic pulsations 3. Global analysis of drift Alfvén-ballooning modes, J. Geophys. Res., 101, 15 441-15 456, doi:10.1029/96JA00494, 1996.

Walker, A. D. M., Greenwald, R. A., Stuart, W. F., and Green, C. A.: STARE auroral radar observations of Pc 5 geomagnetic pulsations, J. Geophys. Res., 84, 3373-3388, 1979.

Warner, M. R. and Orr, D.: Time of flight calculations for high latitude geomagnetic pulsations, Planet. Space Sci., 27, 679-689, doi:10.1016/0032-0633(79)90165-X, 1979.

Ziesolleck, C. W. S., Fraser, B. J., Menk, F. W., and McNabb, P. W.: Spatial characteristics of low-latitude Pc3-4 geomagnetic pulsations, J. Geophys. Res., 98, 197-207, 1993. 